\title{
Dioscorea genus: A Possible Ally in the Sectors of Agriculture, Food Security and as a Source of Inputs for the Pharmaceutical Industry
}

ISSN: 2637-7659

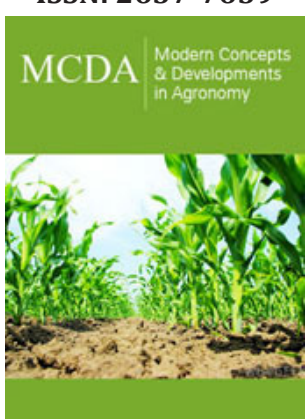

*Corresponding author: Laleska Cesila Rabelo, Agronomic Engineering, Federal University of São Carlos, Araras / SP, Brazil

Submission: 監 August 27, 2021

Published: 僵September 13, 2021

Volume 9 - Issue 3

How to cite this article: Laleska Cesila Rabelo, Luce Maria Brandão Torres. Dioscorea genus: A Possible Ally in the Sectors of Agriculture, Food Security and as a Source of Inputs for the Pharmaceutical Industry. Mod Concep Dev Agrono. 9(3). MCDA. 000713. 2021 DOI: 10.31031/MCDA.2021.09.000713

Copyright@ Laleska Cesila Rabelo. This article is distributed under the terms of the Creative Commons Attribution 4.0 International License, which permits unrestricted use and redistribution provided that the original author and source are credited.
Lalleska Cesilla Rabelo ${ }^{1 *}$ and Luce Maria Brandão Torres ${ }^{2}$

${ }^{1}$ Agronomic Engineering, Federal University of São Carlos, Araras / SP, Brazil

${ }^{2}$ Environmental Research Institute. Department of Infrastructure and Environment, São Paulo/SP, Brazil

\begin{abstract}
Relevance: According to the State of World Food and Nutrition Security, more than 820 million people remain hungry and 2 million are moderately or severely food insecure. The search for new raw materials rich in nutrients and present in Brazilian biodiversity is a new challenge, considering that in the current menu, only 30 species are food sources for $95 \%$ of the world's population. It is expected that new advances in research and new cultivation technologies favor the agriculture of new species with potential use as food and input for the pharmaceutical industry. In this context, we consider that the Dioscorea genus with more than 150 native species, and with some species cultivated and used in food, can be better explored as new matrices rich in nutrients for consumption and as an input for the pharmaceutical industry. The cultivation of Dioscorea species, today is developed with outdated technologies and practiced by small farmers and consequently difficult to maintain production and a delicate harvest requiring new research and technologies to increase productivity and in post-harvest operations.
\end{abstract}

Objective: this review aims to provide data on native species of Dioscorea, as a possible ally in the search for efficiency in the agricultural sector, food safety and source of inputs for the pharmaceutical industry.

Methodology: it was based on data from scientific publications (2000 and 2020) collected in scientific databases using the keywords: Dioscoreaceae, Dioscorea; "igname"; "yam" and "cará".

Result: Dioscorea species native to Brazil have chemical diversity, richness of nutrients and other metabolites, justifying their potential use in food and input for the pharmaceutical industry and other possible applications, as we have shown.

Conclusion: New research approaches and new technologies applied to the study of native species in Brazil can generate knowledge in fighting hunger and in the search for target molecules that can be commercially exploited, promoting innovation in all sectors and environmental preservation as well as sustainable use.

Keywords: Dioscorea; Chemical composition; Pharmacological activities; "Igname, "Cará", "Yam”, Food

\section{Introduction}

From studies initially carried out by Brown in 1819, he identified the family Dioscoreaceae, with the name Dioscoreae. Currently according to Marbberley, 2008, the genus is composed of 850 species distributed in 8 genera, the vast majority belonging to the genus Dioscorea, with approximately $95 \%$ of the species.

The popular name "yam" or "cará" of several Dioscorea species possibly came from the translation of the word "yam" of American origin taken from the French and English colonies of Africa. As for the word yam, by reading the old Brazilian historical documents, it appears to be of indigenous origin. In the Portuguese language, the differentiation of the terms "igname" and "yam" occurs according to the space in which it is inserted, especially in the northeast of Brazil, there is a tendency for the term "igname" to be applied to the large tubers of $D$. cayenensis and the term yam to smaller tubers such as D. alata [1]. As written by Pedralli [2], it is estimated that between 150 and 200 species of Dioscorea occur in Brazil, the only genus of 
the family present in all regions of the country. The main motivation for this review was based on preliminary research on the potential antioxidant effect of Dioscorea dodecaneura leaves collected from the Seedling Nucleus, of the Instituto do Meio Ambiente, SP.

The Northeast region, is the main producing region of "igname" (Dioscorea sp.), using predominantly family labor, with low technological levels, which harms the genus productivity, however, the cultivation of "igname" (Dioscorea sp.) has been growing in recent years [3]. "Dioscorea cayennensis Lam. and D. alata L. with the respective cultivars "da Costa" and "São Tomé" are the species most used in the food crops in Brazil. The first is grown in the Northeast and the second in the North, South and Center South, with São Paulo being the most important producing state [4]. With the increase in the world population, concentrated in urban centers and with access to technological advances, the less favored populations, especially those from less developed countries or regions, connect to these advances in an unequal way, showing the vulnerability of those who cannot keep up globalized growth, unrestrained capitalism and suffer from internal conflicts, due to the governmental instability of their countries, the climate change effects, which cause natural disasters, which will influence in the agricultural and industrial sectors, making it difficult to apply public policies to reduce differences.

Association of those factors, the emergence of new diseases, caused by hunger and environmental problems, such as the lack of ecological succession, due to rampant deforestation, the increase in pollution, caused by the burning of fossil fuels, among others and those that they focus on the current system of production of consumer goods and food, which is antagonistic to the development of food security and, consequently, to the increase in the diversity of raw materials for healthy eating, creating new social, economic, cultural and nutritional challenges, that will benefit health of the world population. According to The State of Food Security and Nutrition in the World, more than 820 million people are still hungry and 2 million people suffer from moderate or severe food insecurity view of the current demand for food security and the challenges to be faced, the introduction of new raw materials rich in nutrients and present in Brazilian biodiversity is fundamental and necessary, considering that 75,000 species have potential use as edible plants and only 200 species have in the food menu, among which, only 30 species reach $95 \%$ of the world population's [5]. The genus Dioscorea can become a great ally to this new self-sustainable and efficient front, based on the potential of their species and its varieties rich in the organic compounds with antioxidant and antimicrobial activities and nutrients with high food value.

However, there is still a scientific ineffectiveness in relation to cultural aspects, bromatological, phytochemical, biochemical, toxicological studies involving all parts of the plant (roots, tubers, leaves, stem and flowers) essential to effective use of these species. "There is an increasing intensification in the search for functional foods, among which the Liliatae family (monocotyledon) stands out, being the species Dioscorea sp of great interest." (Brito et al., 2011).

\section{Material and Methods}

The methodology used in the construction of this review about the potential of the genus Dioscorea was based on data from scientific publications, dated between the years 2000 and 2020 . The data were collected from the scientific bases: Scielo, Google Academico and the Periodicals (Portal CAPES). Where the following keywords were used: Dioscorea; Dioscoreaceae; yam, igname and cará. From the data obtained in the research, an inclusion and exclusion protocol were carried out in order to select and combine the relevant properties related in those reviews.

\section{Result and Discussion}

\section{The Dioscorea genus}

The genus is found in all Brazilian states, as shown in Figure1, having corresponding species in all biomes, in addition to being composed of annual species, which makes it a very important genus in the food, pharmacological and industrial áreas. The diversity of functions in which the genus can be classified, such as: food, nutritional source, bioactive, hormones, biofilm, paper industry, among others, stimulate and justify the need for knowledge of the species.

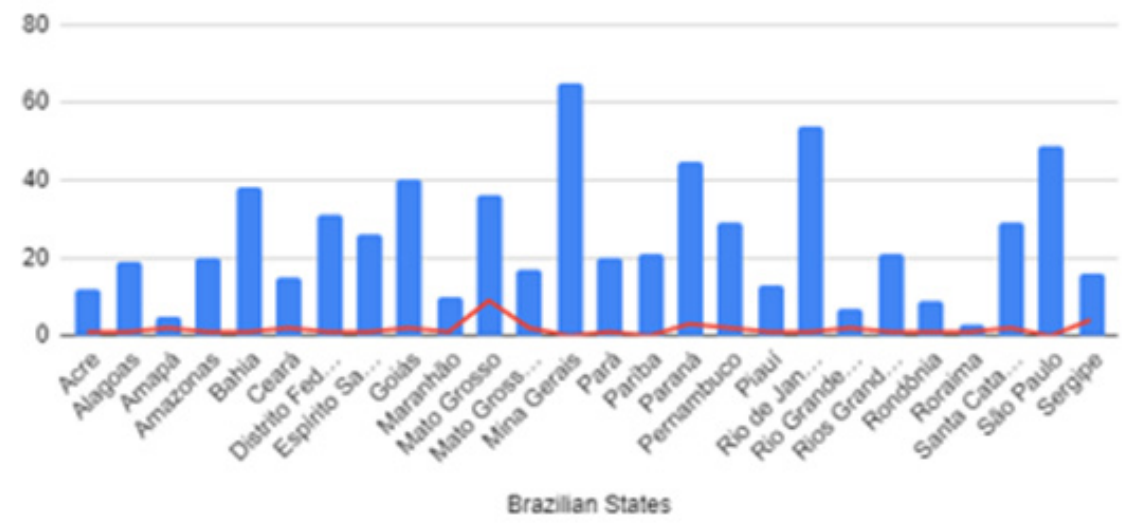

Figure 1: Confirmed occurrence and possible occurrence of the Dioscorea genus. 
In general, Dioscorea genus is composed of creepers, dioecious or monoecious, tuber form or rhizomatous underground system and herbaceous stem. Alternating leaves, sometimes opposite, entire blade lobed, 3-11 primary ribs; petiole with basal genicle sometimes turned into spines. Stem inflorescence raceme simple or with tops, panicle or ear; pistil spike inflorescence; angled, winged racquet; stalk sometimes almost sessile. Unisex, homoclamid flowers; trimer perigone, tube and sepals of variable length. Stemmed solitary flowers, in glomeruli or tops; stamens 3 or 6; staminodes 3 or absent. Sessile pistil flowers; staminodes 3, 6 or absent; free or pyramidal stylets; 3-locular ovary; ova 2 per locule. 3-winged capsule; seeds with a circular or posterior wing [6].

The differentiation between yam and ingame in Brazil is complex and varies from region to region, what is called yam in the Southeast is called yam in the Northeast and vice versa. However, according to the horticultural definition of the species: the yam belongs to the genus Dioscorea, it is a climbing plant that, as it grows, emits branches, and its vegetable consumption product is a tuber, usually with large sizes. The cará, on the other hand, belongs to the genus Colocasia esculenta, composed of herbaceous plants.

\section{Culture productivity}

The Dioscorea genus is composed of plants of relevant importance for man and for the agricultural economy and the pharmaceutical industry. According to Mesquita [7] the main reason for the product of agricultural and industrial exploitation is the tuber, is due to its chemical and phytochemical composition, which showed expressive levels of essential nutrients for human life, such as: carbohydrates, essentials amino acids, pro -vitamin A, pro-vitamin D, vitamins C and B complex, minerals ( $\mathrm{Ca}, \mathrm{P}$ and Fe). Therefore, the active organic compounds that relate these pharmacological effects, which guarantee its use in folk medicine and other with as an input in the synthesis of steroids hormones, as the cortisone. This Dioscorea genus has economic and social value in many areas where cultivation occurs. Some traditional ceremonies are celebrated with yam as the main food, as well as at the Yam Festival in parts of West Africa. Yam production and use is declining in many production areas due to maintenance that demands high demand and the delicate nature of the harvest. Smallholder farmers who produce most of the harvest need access to innovation, which would reduce labor and bring productivity at all levels (on the farm and post-harvest operations) [8].

Dioscorea species are neglected for lack of scientific information that can make their production efficient and profitable for small farmers, since they are the producers of the cultivars, in its majority. The species suffer from a lack of inputs such as: fertilizers, good quality seeds, advances in the breeding sector, which provide a decrease in the incidence of problems related to climate, diseases, pests, planting, post-harvest and with the scarcity of marketing that can enable product insertion for the industrial and family consumer market.

The profitable and commercially and culturally attractive production chain for small producers of the genus and for possible new producers and new trade networks in general, is sustained from substantial information about the difficulties and potential of the same and the lack of information about the genus has numerous consequences that affect this production chain, as an example, one of the main limitations in the production of yams is anthracnose, attributed to the pathogen Colletotrichum (Ascomycota, Glomerellaceae, Sordariomycetes). Anthracnose of yam is characterized by causing damage to the foliage, initially small brown spots appear which then expand causing the complete darkening of the leaf blade. When the disease is very advanced, defoliation is complete. Anthracnose reduces the photosynthetic efficiency of yam plants, affecting yield [9].

\section{Evaluation and chemical composition}

The most studied approaches within the scientific community are the potential of starch in its composition and its applicability to other sources of starch; and the study of plant composition, bromatological, phytochemical and nutritional individual for each species in comparison with other species, with the tuber being taken as the main source of study.

In their studies Feijó et al. [10] obtained the character flour in experiments on laying birds, and the following values were obtained in its chemical composition, 95.54\% dry matter, 2.65\% protein gross; $2.69 \%$ Crude Fiber; $7.45 \%$ Fiber in Neutral Detergent; 3.64\% Fiber in acid detergent; $3.2 \%$ of Mineral Matter; $86.6 \%$ of non-nitrogen extracts; $3730.73 \mathrm{Kcal} / \mathrm{kg}$ of Gross Energy and EM $3489.81 \mathrm{Kcal} / \mathrm{kg}$ the high levels of gross and metabolizable energy obtained characterizes it as an energetic food and with a potential substitute for corn in diets for pigs in the finishing phase. Corroborating the use to feed the flour of yams, Tavares [11], presented data that point the flour of yam-purple as a potential ingredient in the feeding of pigs in the finishing phase, presenting an available energy of $3360 \mathrm{Kcal} / \mathrm{g}$ and good values in centesimal composition.

Within the compositional front of the study, the tuber is the main part of the plant used to discover food functions and possible sources of bioactives, in contrast, the number of studies based on the characterization of the other constituents of the plant is insufficient, which may be, this gap of information, one of the aspects that makes the production of this species forgotten among the production chains, hindering productive efficiency, the flow of production and its residues (leaves, stems and roots). According to Magalhães 2004, in a comparative study between the leaves and the tuber of Dioscorea alata presented the following results for $100 \mathrm{~g}$ on dry basis, respectively, fibers $(42.65 \% ; 22.58 \%)$, proteins (17.52\%; 8.28\%) lipids (4.42\%; 2.09\%), carbohydrates (58.28\%; $76.96 \%)$, phosphorus (22.08\%; 0.63\%), iron $(8.83 \% ; 4.00 \%)$, sodium $(73.82 \% ; 30.70 \%)$ and potassium $(45.87 \% 65.90 \%)$. These data presented by Magalhães demonstrate the potential of the leaves of a species of Dioscorea, which may or may not correspond to other species, leading to the level of food waste that is treated as useless residue of the genus, but which may have social, cultural advantages and economical for small rural producers. 
The research carried out by Souza Gomes, C.; Pacheco Ferreira, L.; dos Reis Nunes, C.; Teixeira Azevedo, F. (2014) covers the perspective of the post-harvest, of the forms of commercialization, characterization of the constituents of the tuber, and quantitatively, the differences in the physical-chemical composition of the yams (Dioscorea Sp.) In natura and after cooking process (15 minutes and 30 minutes), which obtained significant values, respectively, in the percentage of: vitamin $C(8.6 \pm 0.01 ; 3.35 \pm 0.01 ; 2.04 \pm 0.01)$, soluble solids $(7.5 ; 15 ; 15)$ and reducing sugars $(0.42 \pm 0.01 ; 0.69 \pm 0.01$; $0.57 \pm 0.01$ ). In addition to presenting antioxidant potential in all concentrations tested, there was no variation in the scavenging of free radicals in the fresh samples and there was variation in the samples that were cooked, as shown in graphic 2, done by the researchers (Figure 2).

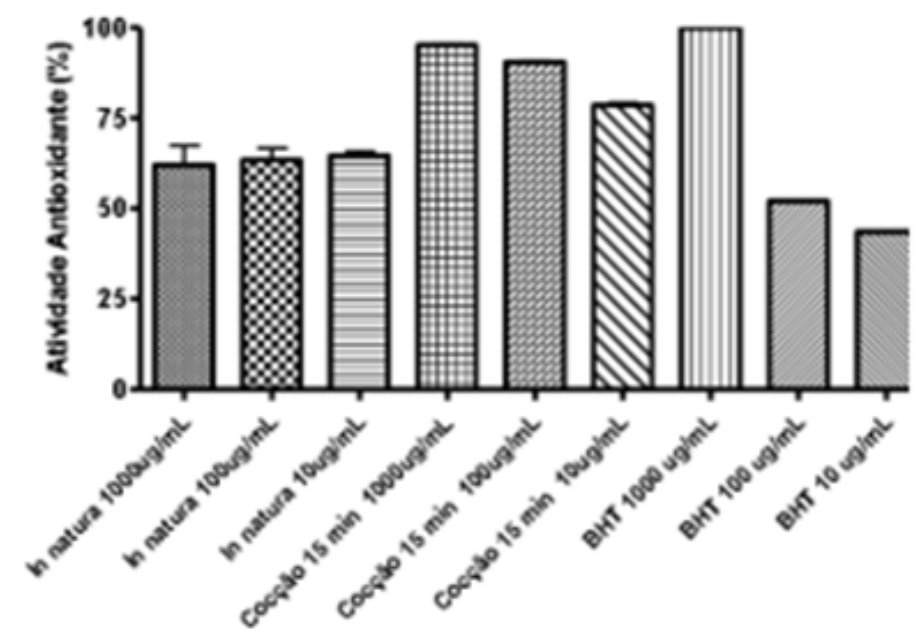

Figure 2: Percentage of antioxidant activity of the yam sample in natura and after cooking process for 15 minutes.

\section{Potentialities of the Dioscorea genus}

The growing demand by a portion of the population, in changing the "normal" consumption of all goods, involves the way you buy, defining you politically, socially, culturally and educationally, placing in the agenda how what is being acquired was generated, if there were and there are environmental impacts, if they are fostered by justice and equality, if it is organic, conventional, agroecological, if it was tested on animals, if it is produced from animals, among so many other struggles for changes. Which leads to the question: how to generate change from a consolidated system? and the answer to the question whether many "depend", however the most promising path to be followed leads to the search for natural, organic products, with minimal environmental, cultural and social impact. Several plant species are unknown, or neglected, and these neglects may be hiding different paths for change, and the genus Dioscorea is a possible source of neglected innovation.

The association of certain cultures, such as yam, it's commonly associated in a scenario of poverty, misery and low-income communities. This is opposed to the extent that this marginalization of the species (a plant that can be considered "orphans") occurs, even in the face of so many contributions provided by them in maintaining life mainly in human health, food and source of biologically active metabolites. In addition, Brazil, into these species being neglected, threatened with extinction and ignorance, they are increasingly "forgotten" on the table of the rural and urban population [12].
The added value of secondary metabolites or natural products (PN) obtained from plants is increasingly important for the survival of living beings on the planet. However, thousands of native species existing in Brazilian biomes still do not have studies in related areas, such as Botany, Ecology, Physiology, Biochemistry, Chemistry of Natural Products and Agronomy and many other species have been studied, but still in an incipient way in these areas, which it generates ignorance and lack of information for producers, manufacturers of service goods, and consumers.

Dioscorea species have several beneficial properties, in particular, they exercise significant antioxidant activity and have the ability to reduce serum lipid levels in humans [13]. In the Amazon Region, the purple yam (D. trifida) can be consumed in different ways, just as its flour can be added in the development of breads, pies, cakes and porridges. However, research on this tuber is still scarce and consequently its nutritional properties are not known [14].

The genus Dioscorea is of great importance in the pharmaceutical sector for supplying diosgenin, a saponin, isolated for the first time in the genus Dioscorea, by Japanese researchers in 1936. The conversion of diosgenin into its steroidal sapogenin and later changed to progesterone occurred in 1940, and the first industrially exploited species were D. composita and D. floribunda" [15]. Diosgenin is also an input for obtaining on an industrial scale in the production of steroidal drugs, including oral contraceptives, topical hormones, systemic corticosteroids, androgens, estrogens, 
progesterone's, but its availability has been lower than the supply required by the pharmaceutical industry [16]. Hu et al. [17], isolated a saponin from the rhizome of $D$. collettii and tested the in vitro cytotoxicity in cancer cell lines, the saponin furostanol showed cytotoxic activity against acute human leukemia, besides presenting potential for colon cancer cure.

Studies carried out in China describe that the presence of physiologically active components such as polysaccharides, saponins and mucins in purple yam are used as antitussive, antidiarrheal and hypoglycemic, as they present satisfactory effects of the lyophilized powder product, and also act as a good antiinflammatory natural (Miyoshi et al., 2011; Park et al., 2013).

The two species Dioscorea alata and Dioscorea cayennensis produce tubers, popularly known as yam or yam, respectively, they are the most well-known and cultivated species in traditional farmers' fields in several states in Brazil. Cultivation is widespread in the Amazon, as it is present in the diet of the local population, and its use associated with these tubers has a high nutritional value, due to the high concentrations of carbohydrates, proteins, phosphorus, calcium, iron and vitamins B1 and B2 [18]. According to Zárete et al. [19], Dioscorea alata can be used by the food industry, because it has several characteristics and similarities with cassava and its starch, both in flavor and texture. According to Leonel et al. [20], among the species studied, Dioscorea sp. showed higher yield per area (6.1ton/ha) in starch production.

The raw tuber of Dioscorea piperifolia is considered to be toxic, but it becomes edible after being subjected to long cooking. Tuberculous rhizomes are also used in food after prolonged cooking. In folk medicine, leaves are used as an emollient. However, the biological potential of this species is nonexistent in the literature [15]. According to experiments carried out by Lopes [21], the extracts in hexane, dichloromethane (DCM), ethyl acetate (AcOEt), and n-butanol (n-But) from the leaves of D. piperifolia, when subjected to several tests using the agar diffusion, to evaluate the ability to inhibit the growth of pathogenic microorganisms, showed positive results, inhibiting the growth of Escherichia coli and Cryptococcus neoformans [22-32]. The results also showed that the relationship between the microbicide/micro biostatic effect of the extracts showed effects, except for E. coli butanol extract and methicillin-resistant Staphylococcus aureus (MRSA) in the extractions with DCM and AcOEt of the tubers [33-40] (Table 1).

Table 1: Species of the Dioscorea occurring in Brazil according to Flora do Brasil 2020.

\begin{tabular}{|c|c|c|}
\hline $\begin{array}{l}\text { Scientific and Popular } \\
\text { Name }\end{array}$ & Origin, Possible Endemism and Occurrence in Brazil & Reference \\
\hline D. acanthogene Rusby & $\begin{array}{l}\text { Native. Endemic of Brazil. Confirmed occurrence: Bahia; Acre; Rondônia; To- } \\
\text { cantins; Goiás; Mato Grosso; Mato Grosso do Sul; Minas Gerais. }\end{array}$ & $\begin{array}{l}\text { Rusby, Bull. New York Bot. Gard., 6: 492, } \\
1910 .\end{array}$ \\
\hline $\begin{array}{l}\text { D. adenantha Uline ex R. } \\
\text { Knuth }\end{array}$ & Native. Endemic of Brazil. Confirmed occurrence: Minas Gerais. & $\begin{array}{l}\text { Uline ex R. Knuth, Das Pflanzenreich IV, } \\
\text { 43: 79, } 1924 .\end{array}$ \\
\hline D. aesculifolia $\mathrm{R}$. Knuth & $\begin{array}{l}\text { Native. Endemic of Brazil. Confirmed occurrence: Mato Grosso do Sul; São } \\
\text { Paulo. }\end{array}$ & $\begin{array}{c}\text { Knuth, R., Pflanzenr. (Engler), IV, 43: } \\
170,1924\end{array}$ \\
\hline D. alata L. & $\begin{array}{c}\text { Cultivada. Endemic to the Asian Continent. Confirmed occurrence: Amazonas; } \\
\text { Rondônia; Roraima; Maranhão; Distrito Federal; Mato Grosso; Minas Gerais; } \\
\text { Rio de Janeiro; São Paulo; Paraná. Possible ocurrence: Acre; Amapá; Alagoas; } \\
\text { Ceará }\end{array}$ & Linnaeus, C., Sp. Pl: 1033, 1753. \\
\hline D. amaranthoides C.Presl & $\begin{array}{l}\text { Native. Confirmed occurrence: Acre; Amazonas; Pará; Rondônia; Bahia; Distrito } \\
\text { Federal; Goiás; Mato Grosso do Sul; Mato Grosso; Minas Gerais; São Paulo Possi- } \\
\text { ble ocurrence: Amapá; Paraná. }\end{array}$ & $\begin{array}{l}\text { Knuth, R., Notizbl. Königl. Bot. Gart. } \\
\text { Berlin, 7: 215, } 1917 .\end{array}$ \\
\hline $\begin{array}{l}\text { D. amazonum Mart. ex } \\
\text { Griseb. }\end{array}$ & $\begin{array}{l}\text { Native. Confirmed occurrence: Acre; Amazonas; Amapá; Pará; Rondônia; Rorai- } \\
\text { ma; Mato Grosso. }\end{array}$ & $\begin{array}{l}\text { Knuth, R., Notizbl. Königl. Bot. Gart. } \\
\text { Berlin, 7: 217, } 1917 .\end{array}$ \\
\hline D. anomala Griseb. & Native. Endemic of Brazil. Confirmed occurrence: Goiás; Minas Gerais. & $\begin{array}{l}\text { Grisebach, A.H.R., Fl. bras., 3(1): 31, } \\
\text { 1842. Ano?? }\end{array}$ \\
\hline $\begin{array}{l}\text { D. argyrogyna Uline ex R. } \\
\text { Knuth }\end{array}$ & Native. Endemic of Brazil. Confirmed occurrence: Mato Grosso. & $\begin{array}{l}\text { Knuth, R., Notizbl. Königl. Bot. Gart. } \\
\text { Berlin, 7: 204, } 1917 .\end{array}$ \\
\hline $\begin{array}{l}\text { D. aspera Humb. \& Bonpl. } \\
\text { ex Willd. }\end{array}$ & Native. Confirmed occurrence: Minas Gerais; Santa Catarina. & Humb. \& Bonpl., Sp. Pl., 4: 794, 1806. \\
\hline D. asperula Pedralli & $\begin{array}{c}\text { Native. Endemic of Brazil. Confirmed occurrence: Distrito Federal; Goiás; Minas } \\
\text { Gerais. }\end{array}$ & Pedralli., G.., Napaea, 8: 29, 1992. \\
\hline D. bahiensis R. Knuth & Native. Endemic of Brazil. Confirmed occurrence: Bahia & $\begin{array}{l}\text { Knuth, R., Pflanzenr. (Engler), IV, 43: } \\
\text { 351, } 1924 .\end{array}$ \\
\hline $\begin{array}{l}\text { D. basiclavicaulis Rizzini \& } \\
\text { A. Mattos }\end{array}$ & $\begin{array}{c}\text { Native. Endemic of Brazil. Confirmed occurrence: Bahia; Espírito Santo; Minas } \\
\text { Gerais }\end{array}$ & $\begin{array}{l}\text { Rizzini, C.T. \& Mattos-Filho, A., Revista } \\
\text { Brasil. Biol., 46: 317, } 1986 .\end{array}$ \\
\hline D. beecheyi R. Knuth & Native. Endemic of Brazil. Confirmed occurrence: Santa Catarina & $\begin{array}{l}\text { Knuth, R., Pflanzenr. (Engler), IV, 43: 58, } \\
1924 .\end{array}$ \\
\hline D. beyrichii R. Knuth & Native. Endemic of Brazil. Confirmed occurrence: Rio de Janeiro; Goiás & $\begin{array}{l}\text { Knuth, R., Notizbl. Königl. Bot. Gart. } \\
\text { Berlin, 7: 210, } 1917 .\end{array}$ \\
\hline
\end{tabular}




\begin{tabular}{|c|c|c|}
\hline D. bradei R. Knuth & Native. Endemic of Brazil. Confirmed occurrence: Rio de Janeiro & $\begin{array}{l}\text { Knuth, R., Repert. Spec. Nov. Regni Veg. } \\
\text { Beih., 42: 177, } 1937 .\end{array}$ \\
\hline $\begin{array}{l}\text { D. bulbifera L. } \\
\text { Cará-da-árvore; Cará-moe- } \\
\text { la; Cará-do-ar. }\end{array}$ & $\begin{array}{l}\text { Naturalized. Endemic to Africa, Índia. Confirmed occurrence: Amazonas; Bahia; } \\
\text { Ceará; Maranhão; Pernambuco; Mato Grosso do Sul; Minas Gerais; Rio de Janei- } \\
\text { ro; São Paulo; Paraná. }\end{array}$ & Linnaeus, C., Sp. Pl.: 1033, 1753. \\
\hline $\begin{array}{l}\text { D. bulbotricha Hand. - } \\
\text { Mazz. }\end{array}$ & $\begin{array}{l}\text { Native. Endemic of Brazil. Confirmed occurrence: São Paulo; Paraná; Santa } \\
\text { Catarina }\end{array}$ & $\begin{array}{l}\text { Handel-Mazzetti, H.R.E., Denkschr. } \\
\text { Kaiserl. Akad. Wiss., Wien. } \\
\text { Math.-Naturwiss. Kl., 79: 221, } 1908 .\end{array}$ \\
\hline D. calcarea R. Knuth & Native. Endemic unknown. Confirmed occurrence: Paraná. & $\begin{array}{c}\text { Knuth, R., Pflanzenr. (Engler), IV, 43: 84, } \\
1924 .\end{array}$ \\
\hline D. caldasensis R. Knuth & Native. Endemic of Brazil. Confirmed occurrence: Minas Gerais. & $\begin{array}{l}\text { Knuth, R., Pflanzenr. (Engler), IV, 43: } \\
\text { 112, } 1924 .\end{array}$ \\
\hline $\begin{array}{l}\text { D. campanulata Uline ex R. } \\
\text { Knuth }\end{array}$ & Native. Endemic of Brazil. Confirmed occurrence: Rio de Janeiro. & $\begin{array}{l}\text { Knuth, R., Notizbl. Königl. Bot. Gart. } \\
\text { Berlin, 7: 189, } 1917\end{array}$ \\
\hline $\begin{array}{l}\text { D. campestris Griseb. } \\
\text { Cará-casco-de-c avalo; } \\
\text { Cará-do-campo. }\end{array}$ & $\begin{array}{l}\text { Native. Endemic of Argentina. Confirmed occurrence: Pará; Alagoas; Bahia; } \\
\text { Ceará; Paraíba; Pernambuco; Piauí; Rio Grande do Norte; Sergipe; Distrito } \\
\text { Federal; Goiás; Mato Grosso do Sul; Mato Grosso; Espírito Santo; Minas Gerais; } \\
\text { Rio de Janeiro; São Paulo; Paraná; Rio Grande do Sul; Santa Catarina. }\end{array}$ & $\begin{array}{l}\text { Grisebach, A.H.R., Fl. bras., 3(1): 30, } \\
1842\end{array}$ \\
\hline D. campos-portoi R. Knuth & Native. Endemic of Brazil. Confirmed occurrence: Rio de Janeiro & $\begin{array}{l}\text { Knuth, R., Repert. Spec. Nov. Regni Veg. } \\
\text { Beih., 42: 176, } 1937 .\end{array}$ \\
\hline $\begin{array}{l}\text { D. cayennensis Lam. } \\
\text { Cará-da-costa; Inhame; } \\
\text { Inhame-de-Pern ambuco. }\end{array}$ & $\begin{array}{l}\text { Cultivada. Endemic to Western Africa. Confirmed occurrence: Bahia; Ceará; } \\
\text { Paraíba; Pernambuco; Minas Gerais; Rio de Janeiro. }\end{array}$ & Lamarck, J.-B., Encycl. Biol., 3: 233, 1789 \\
\hline D. ceratandra R. Knuth & $\begin{array}{l}\text { Native. Endêmica of México. Confirmed occurrence: Tocantins; Distrito Federal; } \\
\text { Goiás; Minas Gerais; Rio de Janeiro; São Paulo; Paraná; Santa Catarina. Possible } \\
\text { ocurrence: Ceará. }\end{array}$ & $\begin{array}{l}\text { Knuth, R., Notizbl. Königl. Bot. Gart. } \\
\text { Berlin, 7: 207, } 1917 .\end{array}$ \\
\hline $\begin{array}{l}\text { D. chondrocarpa Griseb. } \\
\text { Cará-espinho; Cipó-jacaré; } \\
\text { Inhame-de-espi nho. }\end{array}$ & $\begin{array}{l}\text { Native. Confirmed occurrence: Acre; Amazonas; Pará; Rondônia; Tocantins; Ala- } \\
\text { goas; Bahia; Paraíba; Distrito Federal; Goiás; Mato Grosso do Sul; Mato Grosso; } \\
\text { Espírito Santo; Minas Gerais; Rio de Janeiro, São Paulo; Paraná; Rio Grande do } \\
\text { Sul; Santa Catarina. Possible ocurrence: Pernambuco; Sergipe. }\end{array}$ & $\begin{array}{l}\text { Grisebach, A.H.R., Fl. bras., 3(1): 34, } \\
\qquad 1842 .\end{array}$ \\
\hline D. cienegensis R. Knuth & Native. Confirmed occurrence: Paraná; Rio Grande do Sul. & $\begin{array}{l}\text { Knuth, R., Notizbl. Bot. Gart. Ber- } \\
\text { lin-Dahlem, 7: 200, } 1917 .\end{array}$ \\
\hline $\begin{array}{l}\text { D. cinnamomifolia Hook. } \\
\text { Cará-assu; Cará- de-índio; } \\
\text { Cará-peluda. }\end{array}$ & $\begin{array}{l}\text { Native. Endemic of Brazil. Confirmed occurrence: Alagoas; Bahia; Paraíba; Per- } \\
\text { nambuco; Distrito Federal; Goiás; Espírito Santo; Minas Gerais; Rio de Janeiro; } \\
\text { São Paulo; Paraná; Santa Catarina. Possible ocurrence: Rio Grande do Norte; } \\
\text { Sergipe. }\end{array}$ & Hooker, Bot. Mag., 55: t. 2825, 1828. \\
\hline $\begin{array}{l}\text { D. claussenii Uline ex R. } \\
\text { Knuth }\end{array}$ & Native. Endemic of Brazil. Confirmed occurrence: Goiás; Minas Gerais. & $\begin{array}{l}\text { Knuth, R., Notizbl. Königl. Bot. Gart. } \\
\text { Berlin, 7: 215, } 1917 .\end{array}$ \\
\hline D. commutata R. Knuth & Native. Endemic of Brazil. Confirmed occurrence: Santa Catarina. & $\begin{array}{c}\text { Knuth, R., Pflanzenr. (Engler), IV, 43: 74, } \\
1924 .\end{array}$ \\
\hline D. compacta D. Araújo & Native. Endemic of Brazil. Confirmed occurrence: Tocantins. & $\begin{array}{l}\text { Araújo, D., Antar, G.M. \& Lombardi, J.A., } \\
\text { Kew Bull., 71:27, } 2016 .\end{array}$ \\
\hline D. contracta R. Knuth & Native. Endemic of Brazil. Confirmed occurrence: Minas Gerais. & $\begin{array}{l}\text { Knuth, R., Notizbl. Königl. Bot. Gart. } \\
\text { Berlin, 7: 190, } 1917 .\end{array}$ \\
\hline D. coronata Hauman & $\begin{array}{l}\text { Native. Confirmed occurrence: Paraíba; Pernambuco; Sergipe; Rio de Janeiro; } \\
\text { São Paulo; Paraná; Rio Grande do Sul; Santa Catarina. }\end{array}$ & $\begin{array}{l}\text { Hauman, L.L., Anales Mus. Nac. Hist. Nat. } \\
\text { Buenos Aires, 27: 480, } 1916\end{array}$ \\
\hline D. corumbensis R. Knuth & $\begin{array}{l}\text { Native. Endemic of Brazil. Confirmed occurrence: Tocantins; Maranhão; Distri- } \\
\text { to Federal; Goiás; Mato Grosso do Sul. }\end{array}$ & $\begin{array}{l}\text { Knuth, R., Pflanzenr. (Engler), IV, 43: } \\
\text { 241, } 1924 .\end{array}$ \\
\hline D. crotalariifolia Uline & Native. Confirmed occurrence: Amazonas; Pará; Amapá; Mato Grosso. & $\begin{array}{l}\text { Uline, Nat. Pflanzenfam. [Engler \& } \\
\text { Prantl], Nachtr. 1: 85, } 1897 .\end{array}$ \\
\hline D. curitybensis R. Knuth & Native. Endemic of Brazil. Confirmed occurrence: Paraná & $\begin{array}{l}\text { Knuth, R., Pflanzenr. (Engler), IV, 43: } \\
\text { 113, } 1924 .\end{array}$ \\
\hline D. cuyabensis R.Knuth & Native. Endemic of Brazil. Confirmed occurrence: Mato Grosso. & $\begin{array}{l}\text { Knuth, R., Notizbl. Königl. Bot. Gart. } \\
\text { Berlin, 7: 216, } 1917 .\end{array}$ \\
\hline $\begin{array}{l}\text { D. cynanchifolia Griseb. } \\
\text { Cará; Cará-roxa. }\end{array}$ & $\begin{array}{l}\text { Native. Endemic of Brazil. Confirmed occurrence: Distrito Federal; Goiás; } \\
\text { Espírito Santo; MInas Gerais; Rio de Janeiro; São Paulo. }\end{array}$ & $\begin{array}{l}\text { Grisebach, A.H.R., Vidensk. Meddel. } \\
\text { Dansk Natuhist. Foren. Kjøbenhavn, } \\
\text { 1875: 156, } 1875 .\end{array}$ \\
\hline $\begin{array}{l}\text { D. debilis Uline ex RD. } \\
\text { Knuth }\end{array}$ & $\begin{array}{l}\text { Native. Endemic of Brazil. Confirmed occurrence: Bahia; Minas Gerais. Possible } \\
\text { ocurrence: Tocantins; Goiás. }\end{array}$ & $\begin{array}{l}\text { Knuth, R., Notizbl. Königl. Bot. Gart. } \\
\text { Berlin, 7: 210, } 1917 .\end{array}$ \\
\hline
\end{tabular}




\begin{tabular}{|c|c|c|}
\hline D. deflexa Griseb. & $\begin{array}{l}\text { Native. Endemic of Brazil. Confirmed occurrence: Minas Gerais. Possible ocur- } \\
\text { rence: Mato Grosso. }\end{array}$ & $\begin{array}{l}\text { Grisebach, A.H.R., Vidensk. Meddel. } \\
\text { Dansk Natuhist. Foren. Kjøbenhavn, } \\
\text { 1875: 157, } 1875 .\end{array}$ \\
\hline D. delicata R. Knuth & $\begin{array}{l}\text { Native. Endemic of Brazil. Confirmed occurrence: São Paulo; Paraná; Santa } \\
\text { Catarina. }\end{array}$ & $\begin{array}{l}\text { Engler, H.G.A. (ed.), Pflanzenr. (Engler), } \\
\text { IV, 43: 61, } 1924 .\end{array}$ \\
\hline $\begin{array}{l}\text { D. demourae Uline ex R. } \\
\text { Knuth }\end{array}$ & $\begin{array}{l}\text { Native. Confirmed occurrence: Bahia; Pernambuco. Distrito Federal; Minas } \\
\text { Gerais; Rio de Janeiro; São Paulo; Paraná; Rio Grande do Sul; Santa Catarina. }\end{array}$ & $\begin{array}{l}\text { Knuth, R., (Notizbl. Bot. Gart. Ber- } \\
\text { lin-Dahlem, 7: 199, } 1917 .\end{array}$ \\
\hline D. diamantinensis R. Knuth & $\begin{array}{l}\text { Native. Endemic of Brazil. Confirmed occurrence: Minas Gerais. Possible occur- } \\
\text { rence: Mato Grosso. }\end{array}$ & $\begin{array}{l}\text { Knuth, R., Pflanzenr. (Engler), IV, 43: } \\
\text { 231, } 1924 .\end{array}$ \\
\hline $\begin{array}{l}\text { D. dodecaneura Vell. } \\
\text { Cará-do-mato; Caratinga; } \\
\text { Caratinga-roxa. }\end{array}$ & $\begin{array}{l}\text { Native. Confirmed occurrence: Acre; Amazonas; Pará; Rondônia; Alagoas; Bahia; } \\
\text { Ceará; Paraíba; Pernambuco; Piauí; Rio Grande do Norte; Sergipe; Distrito Fed- } \\
\text { eral; Goiás; Mato Grosso do Sul; Mato Grosso; Espírito Santo; Minas Gerais; Rio } \\
\text { de Janeiro; São Paulo; Paraná; Rio Grande do Sul; Santa Catarina }\end{array}$ & $\begin{array}{l}\text { Vellozo, J.M.C., Fl. Flumin. Icon., 10: t. } \\
\text { 123, } 1831 .\end{array}$ \\
\hline $\begin{array}{l}\text { D. dumetosa Uline ex R. } \\
\text { Knuth }\end{array}$ & $\begin{array}{l}\text { Native. Endemic of Brazil. Confirmed occurrence: Tocantins; Piauí; Mato } \\
\text { Grosso. }\end{array}$ & $\begin{array}{l}\text { Knuth, R., Notizbl. Königl. Bot. Gart. } \\
\text { Berlin, 7: 209, } 1917 .\end{array}$ \\
\hline D. epistephioides Taub. & Native. Endemic of Brazil. Confirmed occurrence: Goiás. & Taubert, Bot. Jahrb. Syst., 21: 425, 1896 \\
\hline $\begin{array}{l}\text { D. flabellispina R. Couto \& J. } \\
\text { M. A. Braga }\end{array}$ & $\begin{array}{l}\text { Native. Endemic of Brazil. Confirmed occurrence: Rio de Janeiro. Possible } \\
\text { ocurrence: Espírito Santo. }\end{array}$ & $\begin{array}{l}\text { Couto, R. S., Lopes, R. C. \& Braga, J. M. A., } \\
\text { Phytotaxa, 231(1): 89-94, } 2015 .\end{array}$ \\
\hline D. fodinarum Kunth & $\begin{array}{l}\text { Native. Endemic of Brazil. Confirmed occurrence: Bahia; Goiás; Mato Grosso; } \\
\text { Minas Gerais; Rio de Janeiro; São Paulo. }\end{array}$ & Kunth, Enum. Pl. [Kunth], 5: 405, 1850 \\
\hline D. fractiflexa R. Knuth & Native. Endemic of Brazil. Confirmed occurrence: Paraná. & $\begin{array}{l}\text { Knuth, R., Pflanzenr. (Engler), IV, 43: } \\
\text { 235, } 1924 .\end{array}$ \\
\hline D. furcata Griseb. & $\begin{array}{l}\text { Native Confirmed occurrence: Paraná; Rio Grande do sul. Possible occurrence: } \\
\text { Rio de Janeiro; Santa Catarina. }\end{array}$ & Grisebach, A., Fl. bras., 3(1): 45, 1842 \\
\hline D. galiiflora R. Knuth & Native. Endemic of Brazil. Confirmed occurrence: Mato Grosso. & $\begin{array}{l}\text { Knuth, R., Notizbl. Königl. Bot. Gart. } \\
\text { Berlin, 7: 538, } 1921 .\end{array}$ \\
\hline $\begin{array}{l}\text { D. glandulosa (Griseb.) } \\
\text { Kunth }\end{array}$ & $\begin{array}{c}\text { Native. Confirmed occurrence: Amazonas; Pará; Tocantins; Alagoas; Bahia; } \\
\text { Paraíba; Pernambuco; Piauí; Sergipe; Distrito Federal; Goiás; Mato Grosso } \\
\text { do Sul; Mato Grosso; Espírito Santo; Minas Gerais; Rio de Janeiro; São Paulo; } \\
\text { Paraná; Rio Grande do Sul; Santa Catarina }\end{array}$ & Kunth, Enum. Pl. [Kunth], 5: 352, 1850 \\
\hline D. gracilicaulis R. Knuth & Native. Endemic of Brazil. Confirmed occurrence: Goiás; Mato Grosso. & $\begin{array}{l}\text { Knuth, R., Notizbl. Königl. Bot. Gart. } \\
\text { Berlin, 7: 216, } 1917 .\end{array}$ \\
\hline $\begin{array}{l}\text { D. grandiflora Mart. ex } \\
\text { Griseb. }\end{array}$ & $\begin{array}{l}\text { Native Confirmed occurrence: Pará; Goiás; Mato Grosso; Minas Gerais; São } \\
\text { Paulo. }\end{array}$ & $\begin{array}{l}\text { Griesebach, A.H.R., Fl. bras., 3(1): 28, } \\
1842 .\end{array}$ \\
\hline D. grisebachii Kunth & $\begin{array}{l}\text { Native. Endemic of Brazil. Confirmed occurrence: Pará; Tocantins; Bahia Per- } \\
\text { nambuco; Piauí; Sergipe; Distrito Federal; Goiás; Espírito Santo; Minas Gerais; } \\
\text { Rio de Janeiro; São Paulo; Paraná; Rio Grande do Sul; Santa Catarina. }\end{array}$ & Kunth, Enum. Pl. [Kunth], 5: 853, 1850 \\
\hline D. hassleriana Chodat Cará & $\begin{array}{l}\text { Native. Endemic of Brazil. Confirmed occurrence: Bahia; Ceará; Paraíba; Per- } \\
\text { nambuco; Sergipe; Distrito Federal; Goiás; Mato Grosso do Sul; Mato Grosso; } \\
\text { Minas Gerais; Rio de Janeiro; São Paulo; Paraná; Rio Grande do Sul. }\end{array}$ & $\begin{array}{l}\text { Chodat, E., Bull. Herb. Boissier, II, 3: } \\
\text { 1111, } 1903 .\end{array}$ \\
\hline D. heptaneura Vell. & $\begin{array}{l}\text { Native. Endemic of Brazil Confirmed occurrence: Bahia; Minas Gerais; Rio de } \\
\text { Janeiro. }\end{array}$ & C.A.W. Schwacke, 8419, B, (MG), \\
\hline D. huberi R. Knuth & $\begin{array}{l}\text { Native. Endemic of Brazil. Confirmed occurrence: Pará. Possible ocurrence: } \\
\text { Amazonas; Mato Grosso. }\end{array}$ & $\begin{array}{l}\text { Knuth, R., Repert. Spec. Nov. Regni Veg. } \\
\text { Beih., 28: 87, } 1930 .\end{array}$ \\
\hline D. itapirensis R. Knuth & Native. Endemic of Brazil. Confirmed occurrence: São Paulo. & $\begin{array}{l}\text { Knuth, R., Notizbl. Königl. Bot. Gart. } \\
\text { Berlin, 7: 190, } 1917 .\end{array}$ \\
\hline D. itatiaiensis R. Knuth & Native. Endemic of Brazil Confirmed occurrence: Rio de Janeiro. & $\begin{array}{l}\text { Knuth, R., Notizbl. Königl. Bot. Gart. } \\
\text { Berlin, 7:212, } 1917 .\end{array}$ \\
\hline $\begin{array}{l}\text { D. kunthiana Uline ex } \\
\text { R.Knuth }\end{array}$ & $\begin{array}{l}\text { Native. Endemic of Brazil. Confirmed occurrence: Bahia; Minas Gerais; São } \\
\text { Paulo. }\end{array}$ & $\begin{array}{l}\text { Knuth, R., Notizbl. Königl. Bot. Gart. } \\
\text { Berlin, 7: 211, } 1917 .\end{array}$ \\
\hline D. lacerdaei Griseb. & $\begin{array}{l}\text { Native. Endemic of Brazil. Confirmed occurrence: Pará Possible occurrence: } \\
\text { Mato Grosso. }\end{array}$ & Grisebach, A., Fl. bras., 3(1): 31, 1842 \\
\hline $\begin{array}{l}\text { D. laxiflora Mart. ex Griseb. } \\
\text { Cará. }\end{array}$ & $\begin{array}{l}\text { Native. Endemic of Brazil Confirmed occurrence: Acre; Amazonas; Pará; } \\
\text { Alagoas; Bahia; Ceará; Paraíba; Pernambuco; Piauí; Sergipe; Distrito Federal; } \\
\text { Goiás; Mato Grosso; Espírito Santo; Minas Gerais; Rio de Janeiro; São Paulo; } \\
\text { Paraná; Rio Grande do Sul; Santa Catarina. }\end{array}$ & Grisebach, A., Fl. bras., 3(1): 32, 1842 \\
\hline
\end{tabular}




\begin{tabular}{|c|c|c|}
\hline $\begin{array}{l}\text { D. leptobotrys Uline ex R. } \\
\text { Knuth }\end{array}$ & Native. Endemic of Brazil. Confirmed occurrence: São Paulo. & $\begin{array}{l}\text { Knuth, R., Notizbl. Königl. Bot. Gart. } \\
\text { Berlin, 7: 218, } 1917 .\end{array}$ \\
\hline $\begin{array}{l}\text { D. leptostachya Gardner } \\
\text { Cará- amarelo }\end{array}$ & $\begin{array}{l}\text { Native. Endemic of Brazil. Confirmed occurrence: Tocantins; Alagoas; Bahia; } \\
\text { Ceará; Paraíba; Pernambuco; Piauí; Rio Grande do Norte; Sergipe; Distrito } \\
\text { Federal; Mato Grosso; Espírito Santo; Minas Gerais; Rio de Janeiro; São Paulo. }\end{array}$ & Gardner, London J. Bot., 1: 534, 1842 \\
\hline $\begin{array}{l}\text { D. lindmanii Uline ex R. } \\
\text { Knuth }\end{array}$ & Native. Confirmed occurrence: Goiás; Mato Grosso. & $\begin{array}{l}\text { Knuth, R., Notizbl. Königl. Bot. Gart. } \\
\text { Berlin, 7: 190, } 1917 .\end{array}$ \\
\hline D. loefgrenii R. Knuth & Native. Endemic of Brazil. Confirmed occurrence: Minas Gerais. & $\begin{array}{l}\text { Knuth, R., Notizbl. Königl. Bot. Gart. } \\
\text { Berlin, 7: 187, } 1917 .\end{array}$ \\
\hline D. lundii Uline ex R. Knuth & Native. Endemic of Brazil Confirmed occurrence: Rio de Janeiro; São Paulo. & $\begin{array}{l}\text { Knuth, R., Notizbl. Königl. Bot. Gart. } \\
\text { Berlin, 7: 198, } 1917 .\end{array}$ \\
\hline $\begin{array}{l}\text { D. macrantha Uline ex R. } \\
\text { Knuth }\end{array}$ & Native. Endemic of Brazil. Confirmed occurrence: Santa Catarina. & $\begin{array}{l}\text { Knuth, R., Notizbl. Königl. Bot. Gart. } \\
\text { Berlin, 7: 198, } 1917 .\end{array}$ \\
\hline D. macrothyrsa Uline & Native. Endemic of Brazil. Confirmed occurrence: Bahia. & $\begin{array}{l}\text { Uline, Nat. Pflanzenfam. [Engler \& } \\
\text { Prantl], Nachtr. 1: 87, } 1897 .\end{array}$ \\
\hline $\begin{array}{l}\text { D. Maianthemoide s Uline } \\
\text { ex R. Knuth }\end{array}$ & $\begin{array}{l}\text { Native. Endemic of Brazil. Confirmed occurrence: Distrito Federal; Goiás; Minas } \\
\text { Gerais. }\end{array}$ & $\begin{array}{l}\text { Knuth, R., Notizbl. Königl. Bot. Gart. } \\
\text { Berlin, 7: 188, } 1917 .\end{array}$ \\
\hline D. mantiqueirensis R. Knuth & Native. Endemic of Brazil. Confirmed occurrence: Espírito Santo. & $\begin{array}{l}\text { Knuth, R., Notizbl. Königl. Bot. Gart. } \\
\text { Berlin, 7: 192, } 1917 .\end{array}$ \\
\hline $\begin{array}{l}\text { D. margarethia G. M. Bar- } \\
\text { roso et al. }\end{array}$ & Native. Endemic of Brazil. Confirmed occurrence: Rio de Janeiro. & $\begin{array}{l}\text { Barroso, G.M. et al., Loefgrenia, 49: 2, } \\
1970 .\end{array}$ \\
\hline D. marginata Griseb. & $\begin{array}{l}\text { Native. Confirmed occurrence: Amazonas; Pará; Tocantins; Alagoas; Bahia; } \\
\text { Maranhão; Paraíba; Pernambuco; Distrito Federal; Goiás; Mato Grosso do Sul; } \\
\text { Mato Grosso; Espírito Santo, Minas Gerais, Rio de Janeiro, São Paulo; Paraná. }\end{array}$ & Grisebach, A., Fl. bras., 3(1): 37, 1842 \\
\hline D. martiana Griseb. & $\begin{array}{l}\text { Native. Endemic of Brazil. Confirmed occurrence: Ceará; Paraíba; Pernambuco; } \\
\text { Mato Grosso; Rio de Janeiro; Paraná. }\end{array}$ & Grisebach, A., Fl. bras., 3(1): 44, 1842 \\
\hline $\begin{array}{l}\text { D. medusae F. Fraga, R. } \\
\text { Couto \& J.M.A. Braga }\end{array}$ & Native. Confirmed occurrence: Espírito Santo. & \\
\hline D. megacarpa Gleason & Native. Confirmed occurrence: Amazonas; Amapá; Pará; Bahia. & $\begin{array}{l}\text { Gleason, Bull. Torrey Bot. Club, 52: 184, } \\
1925\end{array}$ \\
\hline $\begin{array}{l}\text { D. melastomatifolia Uline } \\
\text { ex Prain }\end{array}$ & Native. Confirmed occurrence: Amazonas; Pará; Maranhão; Mato Grosso. & $\begin{array}{l}\text { Prain, D., Bull. Misc. Inform. Kew, 1916: } \\
\text { 194, } 1916 .\end{array}$ \\
\hline D. microcephala Uline & Native. Endemic of Brazil. Confirmed occurrence: Rio Grande do Sul. & $\begin{array}{l}\text { Uline, Nat. Pflanzenfam. [Engler \& } \\
\text { Prantl], Nachtr. 1: 85, } 1897 .\end{array}$ \\
\hline $\begin{array}{l}\text { D. mollis Kunth Den- } \\
\text { te-de-gato. }\end{array}$ & $\begin{array}{l}\text { Native. Endemic of Brazil. Confirmed occurrence: Bahia; Espírito Santo; Minas } \\
\text { Gerais; Rio de Janeiro; São Paulo. Possible occurrence: Distrito Federal; Goiás; } \\
\text { Mato Grosso do Sul; Mato Grosso; Paraná; Rio Grande do Sul; Santa Catarina. }\end{array}$ & Kunth, Enum. Pl. [Kunth], 5: 369, 1850 \\
\hline $\begin{array}{l}\text { D. monadelpha (Kunth) } \\
\text { Griseb. }\end{array}$ & $\begin{array}{l}\text { Native. Endemic of Brazil. Confirmed occurrence: Alagoas; Bahia; Paraíba; } \\
\text { Pernambuco; Espírito Santo; Minas Gerais; Rio de Janeiro; São Paulo; Paraná; } \\
\text { Rio Grande do Sul; Santa Catarina. Possible ocurrence: Sergipe. }\end{array}$ & $\begin{array}{l}\text { Grisebach, A., Vidensk. Meddel. Dansk } \\
\text { Natuhist. oren. Kjøbenhavn, 1875: 164, } \\
1875 .\end{array}$ \\
\hline D. mosqueirensis R. Knuth & Native. Endemic of Brazil. Confirmed occurrence: Pará & $\begin{array}{l}\text { Knuth, R., Repert. Spec. Nov. Regni Veg. } \\
\text { Beih., 29: 92, } 1931 .\end{array}$ \\
\hline $\begin{array}{l}\text { D. mucronata Uline ex R. } \\
\text { Knuth }\end{array}$ & Native. Endemic of Brazil. Confirmed occurrence: Ceará; Pernambuco. & $\begin{array}{l}\text { Knuth, R., Notizbl. Königl. Bot. Gart. } \\
\text { Berlin, 7: 188, } 1917 .\end{array}$ \\
\hline $\begin{array}{l}\text { D. multiflora Mart. ex } \\
\text { Griseb. Inhame-de-espi } \\
\text { nho; Japecanga. }\end{array}$ & $\begin{array}{l}\text { Native. Endemic of Argentina. Confirmed occurrence: Amazonas; Pará; } \\
\text { Tocantins; Alagoas; Bahia; Ceará; Maranhão; Paraíba; Distrito Federal; Goiás; } \\
\text { Mato Grosso do Sul; Mato Grosso; Espírito Santo; Minas Gerais; Rio de Janeiro; } \\
\text { São Paulo; Paraná; Rio Grande do Sul; Santa Catarina. Possible occurrence: } \\
\text { Pernambuco; Piauí; Rio Grande Do Norte; Sergipe. }\end{array}$ & Grisebach, A., Fl. bras., 3(1): 35, 1842 \\
\hline D. multispicata R. Knuth & Native. Confirmed occurrence: Minas Gerais. & $\begin{array}{l}\text { Knuth, R., Meded. Bot. Mus. Herb. Rijks } \\
\text { Univ. Utrecht, 29: 55, } 1916 .\end{array}$ \\
\hline $\begin{array}{l}\text { D. neblinensis Maguire \& } \\
\text { Steyerm. }\end{array}$ & Native. Endemic of Brazil. Confirmed occurrence: Amazonas. & $\begin{array}{l}\text { Maguire \& Steyerm., Mem. New York } \\
\text { Bot. Gard., 51: 108, } 1989 .\end{array}$ \\
\hline D. nuda R. Knuth & $\begin{array}{l}\text { Native. Endemic of Brazil. Confirmed occurrence: São Paulo Possible ocur- } \\
\text { rence: Mato Grosso. }\end{array}$ & $\begin{array}{l}\text { Knuth, R., Pflanzenr. (Engler), IV, 43: } \\
\text { 351, } 1924 .\end{array}$ \\
\hline D. nutans R.knuth & $\begin{array}{l}\text { Native. Endemic of Brazil. Confirmed occurrence: Minas Gerais. Possible ocur- } \\
\text { rence: Mato Grosso. }\end{array}$ & $\begin{array}{l}\text { Knuth, R., Pflanzenr. (Engler), IV, 43: } \\
\text { 182, } 1924 .\end{array}$ \\
\hline
\end{tabular}




\begin{tabular}{|c|c|c|}
\hline $\begin{array}{l}\text { D. olfersiana Klotzsch ex } \\
\text { Griseb. Cará-do-sapo }\end{array}$ & $\begin{array}{l}\text { Native. Endemic of Brazil. Confirmed occurrence: Bahia; Pernambuco; Distrito } \\
\text { Federal; Minas Gerais; Rio de Janeiro; São Paulo; Paraná; Santa Catarina. }\end{array}$ & Grisebach, A., Fl. bras., 3(1): 38, 1842 \\
\hline D. oppositiflora Griseb. & Native. Endemic of Brazil. Confirmed occurrence: Rio de Janeiro. & Grisebach, A., Fl. bras., 3(1): 46, 1842 \\
\hline D. organensis R. Knuth & Native. Endemic of Brazil. Confirmed occurrence: Rio de Janeiro. & $\begin{array}{l}\text { Engler, H.G.A. (ed.), Pflanzenr. (Engler), } \\
\text { IV, 43: 106, } 1924 .\end{array}$ \\
\hline $\begin{array}{l}\text { D. orthogoneura Uline ex } \\
\text { Hochr. }\end{array}$ & $\begin{array}{l}\text { Native. Confirmed occurrence: Tocantins; Bahia; Ceará; Maranhão; Pernambu- } \\
\text { co; Piauí; Rio Grande do Norte; Sergipe; Distrito Federal; Goiás; Mato Grosso do } \\
\text { Sul; Mato Grosso; Minas Gerais. }\end{array}$ & $\begin{array}{l}\text { Hochreutiner, B.P.G., Bull. New York Bot. } \\
\text { Gard., 6: 267, } 1910 .\end{array}$ \\
\hline $\begin{array}{l}\text { D. ovata Vell. Cará-de-pe- } \\
\text { dra; Inhame-bravo }\end{array}$ & $\begin{array}{c}\text { Native. Endemic of Brazil. Confirmed occurrence: Alagoas; Bahia; Ceará; } \\
\text { Paraíba; Pernambuco; Piauí; Rio Grande do Norte; Sergipe; Distrito Federal; } \\
\text { Goiás;Espírito Santo; Minas Gerais; Rio de Janeiro; São Paulo; Paraná; Rio } \\
\text { Grande do Sul; Santa Catarina. }\end{array}$ & $\begin{array}{l}\text { Vellozo, J.M.C., Fl. Flumin. Icon., 10: t. } \\
\text { 117, } 1831 .\end{array}$ \\
\hline D. pallidinervia R. Knuth & Native. Endemic of Brazil. Confirmed occurrence: Paraná. & $\begin{array}{c}\text { Knuth, R., Pflanzenr. (Engler), IV, 43: 75, } \\
1924 .\end{array}$ \\
\hline $\begin{array}{l}\text { D. pedalis (Uline ex R. } \\
\text { Knuth) R. Couto \& J. M. A. } \\
\text { Braga }\end{array}$ & Native. Endemic of Brazil Confirmed occurrence: Rio de Janeiro. & $\begin{array}{l}\text { Couto et al., Systematic Botany, Vol. } \\
\text { 39(3), } 2014 .\end{array}$ \\
\hline D. perdicum Taub. & Native. Endemic of Brazil. Confirmed occurrence: Minas Gerais; Rio de Janeiro. & $\begin{array}{l}\text { Taubert, Bot. Jahrb. Syst., 15(34): 13, } \\
1892\end{array}$ \\
\hline D. piauhyensis R. Knuth & Native. Endemic of Brazil. Confirmed occurrence: Piauí. & $\begin{array}{c}\text { Knuth, R., Pflanzenr. (Engler), IV, 43: 64, } \\
1924 .\end{array}$ \\
\hline $\begin{array}{l}\text { D. pilosiuscula Betero ex } \\
\text { Spreng. }\end{array}$ & Native. Confirmed occurrence: Amazonas; Amapá; Pará. & Sprengel, Syst. Veg., 2: 152, 1982. \\
\hline $\begin{array}{l}\text { D. piperifolia Humb. \& } \\
\text { Bonpl. ex Willd. Cará; Cará- } \\
\text { do-mato; Cosco-de-burro; } \\
\text { Inhame-bravo. }\end{array}$ & $\begin{array}{l}\text { Native. Confirmed occurrence: Acre; Amazonas; Pará; Rondônia; Tocantins; } \\
\text { Alagoas; Bahia; Ceará; Maranhão; Paraíba; Pernambuco; Piauí; Sergipe; Distrito } \\
\text { Federal; Goiás; Mato Grosso do Sul; Mato Grosso; Espírito Santo; Minas Gerais; } \\
\text { Rio de Janeiro; São Paulo; Paraná; Rio Grande do Sul; Santa Catarina }\end{array}$ & Humb. \& Bonpl., Sp. Pl., 4: 795, 1806. \\
\hline $\begin{array}{l}\text { D. planistipulosa Uline ex } \\
\text { R. Knuth }\end{array}$ & $\begin{array}{l}\text { Native. Endemic of Brazil. Confirmed occurrence: Bahia; Piauí; Rio de Janeiro; } \\
\text { São Paulo. }\end{array}$ & $\begin{array}{l}\text { Knuth, R., Notizbl. Königl. Bot. Gart. } \\
\text { Berlin, 7: 204, } 1917 .\end{array}$ \\
\hline D. pohlii Griseb. & $\begin{array}{l}\text { Native. Endemic of Brazil. Confirmed occurrence: Acre; Maranhão; Goiás; Mato } \\
\text { Grosso. }\end{array}$ & Grisebach, A., Fl. bras., 3(1): 35, 1842 \\
\hline $\begin{array}{l}\text { D. polygonoides Humb. \& } \\
\text { Bonpl. ex Willd. caratin- } \\
\quad \text { ga-branca }\end{array}$ & $\begin{array}{l}\text { Native. Confirmed occurrence: Tocantins; Alagoas; Bahia; Ceará; Paraíba; } \\
\text { Pernambuco; Piauí; Rio Grande do Norte; Sergipe; Distrito Federal; Goiás; Mato } \\
\text { Grosso; Espírito Santo; Minas Gerais; Rio de Janeiro; São Paulo; Paraná; Santa } \\
\text { Catarina. }\end{array}$ & Humb. \& Bonpl., Sp. Pl., 4: 795, 1806. \\
\hline D. psammophila R. Knuth & Native. Endemic of Brazil. Confirmed occurrence: Bahia. & $\begin{array}{l}\text { Knuth, R., Repert. Spec. Nov. Regni Veg. } \\
\text { Beih., 38: 120, } 1935 .\end{array}$ \\
\hline $\begin{array}{l}\text { D. pseudomacro capsa G.M. } \\
\text { Barroso et al. }\end{array}$ & Native. Endemic of Brazil. Confirmed occurrence: Rio de Janeiro. & $\begin{array}{l}\text { Barroso, G.M. et al., Revista Brasil. Biol., } \\
\text { 31: 309, } 1971 .\end{array}$ \\
\hline D. pumilio Griseb. & Native. Endemic of Brazil. Confirmed occurrence: Rio de Janeiro. & $\begin{array}{l}\text { Grisebach, A., Vidensk. Meddel. Dansk } \\
\text { Natuhist. Foren. Kjøbenhavn, 1875: 162, } \\
\text { 1875. }\end{array}$ \\
\hline D. puncticulata R. Knuth & Native. Endemic of Brazil. Confirmed occurrence: Paraná. & $\begin{array}{c}\text { Knuth, R., Pflanzenr. (Engler), IV, 43: 55, } \\
1924 .\end{array}$ \\
\hline $\begin{array}{l}\text { D. regnellii Uline ex R. } \\
\text { Knuth }\end{array}$ & $\begin{array}{l}\text { Native. Endemic of Brazil. Confirmed occurrence: Minas Gerais; Rio de Janeiro; } \\
\text { São Paulo. Possible occurrence: Mato Grosso; Santa Catarina. }\end{array}$ & $\begin{array}{l}\text { Knuth, R., Notizbl. Königl. Bot. Gart. } \\
\text { Berlin, 7: 214, } 1917 .\end{array}$ \\
\hline D. riedelii $\mathrm{R}$. Knuth & Native. Endemic of Brazil. Confirmed occurrence: Mato Grosso. & $\begin{array}{l}\text { Knuth, R., Notizbl. Königl. Bot. Gart. } \\
\text { Berlin, 7: 213, } 1917 .\end{array}$ \\
\hline $\begin{array}{l}\text { D. riparia Kunth \& M.R. } \\
\text { Schomb. }\end{array}$ & $\begin{array}{l}\text { Native. Endemic of Brazil. Confirmed occurrence: Acre; Amazonas; Goiás; Mato } \\
\text { Grosso. Possible occurrence: Rondônia; Bahia. }\end{array}$ & $\begin{array}{l}\text { Kunth, C.S., Enum. Pl. [Kunth], 5: 364, } \\
1850 .\end{array}$ \\
\hline D. rumicoides Griseb. & $\begin{array}{l}\text { Native. Endemic of Brazil. Confirmed occurrence: Paraíba; Sergipe; Distrito } \\
\text { Federal; Goiás; Mato Grosso; Minas Gerais; Rio de Janeiro; São Paulo; Paraná; } \\
\text { Santa Catarina }\end{array}$ & $\begin{array}{l}\text { Griesebach, A.H.R., Fl. bras., 3(1): 42, } \\
\qquad 1842 .\end{array}$ \\
\hline D. sabarensis R. Knuth & Native. Endemic of Brazil. Confirmed occurrence: Minas Gerais. & $\begin{array}{l}\text { Knuth, R., Pflanzenr. (Engler), IV, 43: } \\
\qquad 241,1924 .\end{array}$ \\
\hline D. sanpaulensis R. Knuth & Native. Endemic of Brazil. Confirmed occurrence: São Paulo. & $\begin{array}{c}\text { Knuth, R., Pflanzenr. (Engler), IV, 43: 57, } \\
1924 .\end{array}$ \\
\hline
\end{tabular}




\begin{tabular}{|c|c|c|}
\hline D. santosensis R. Knuth & Native. Endemic of Brazil. Confirmed occurrence: São Paulo. & $\begin{array}{l}\text { Knuth, R., Repert. Spec. Nov. Regni Veg. } \\
\text { Beih., 21: 77, } 1925 .\end{array}$ \\
\hline $\begin{array}{l}\text { D. scabra Humb. \& Bonpl. } \\
\quad \text { ex Willd. cará }\end{array}$ & $\begin{array}{l}\text { Native. Endemic of Brazil. Confirmed occurrence: Bahia; Goiás; Mato Grosso; } \\
\text { Espírito Santo; Minas Gerais; São Paulo; Paraná; Rio Grande do Sul; Santa Cata- } \\
\text { rina. Possible ocurrence: Pará; Maranhão; Mato Grosso do Sul. }\end{array}$ & Humb. \& Bonpl., Sp. Pl., 4: 794, 1806. \\
\hline $\begin{array}{l}\text { D. schwackei Uline ex R. } \\
\text { Knuth }\end{array}$ & Native. Endemic of Brazil. Confirmed occurrence: Minas Gerais. & $\begin{array}{l}\text { Knuth, R., Notizbl. Königl. Bot. Gart. } \\
\text { Berlin, 7: 195, } 1917 .\end{array}$ \\
\hline D. secunda R. Knuth & Native. Endemic of Brazil. Confirmed occurrence: São Paulo. & $\begin{array}{l}\text { Knuth, R., Pflanzenr. (Engler), IV, 43: } \\
\text { 350, } 1924 .\end{array}$ \\
\hline $\begin{array}{l}\text { D. sellowiana Uline ex R. } \\
\text { Knuth }\end{array}$ & Native. Confirmed occurrence: São Paulo; Rio Grande do Sul & $\begin{array}{l}\text { Knuth, R., Notizbl. Königl. Bot. Gart. } \\
\text { Berlin, 7: 198, } 1917 .\end{array}$ \\
\hline D. septemnervis Vell. & Native. Endemic of Brazil. Confirmed occurrence: Rio de Janeiro. & $\begin{array}{l}\text { Vellozo, J.M.C., Fl. Flumin. Icon., 10: 119, } \\
1831 .\end{array}$ \\
\hline $\begin{array}{l}\text { D. sincorensis R.Knuth cará- } \\
\text { de-pedra }\end{array}$ & $\begin{array}{l}\text { Native. Endemic of Brazil. Confirmed occurrence: Alagoas; Bahia; Pernambuco; } \\
\text { Distrito Federal; Goiás; Mato Grosso do Sul; Espírito Santo; Minas Gerais. }\end{array}$ & $\begin{array}{l}\text { Knuth, R., Notizbl. Königl. Bot. Gart. } \\
\text { Berlin, 7: 186, } 1917 .\end{array}$ \\
\hline $\begin{array}{l}\text { D. sinuata Vell. Cará-bravo } \\
\text { Caratinga-brava }\end{array}$ & $\begin{array}{l}\text { Native. Confirmed occurrence: Tocantins; Alagoas; Bahia; Paraíba; Pernambu- } \\
\text { co; Rio Grande do Norte; Sergipe; Distrito Federal; Goiás; Mato Grosso do Sul; } \\
\text { Espírito Santo; Minas Gerais; Rio de Janeiro; São Paulo; Paraná; Rio Grande do } \\
\text { Sul; Santa Catarina. }\end{array}$ & $\begin{array}{l}\text { Vellozo, J.M.C., Fl. Flumin. Icon., 10: t. } \\
\text { 129, } 1831 .\end{array}$ \\
\hline $\begin{array}{l}\text { D. sphaeroidea R. Couto \& } \\
\text { J.M.A. Braga }\end{array}$ & Native. Endemic of Brazil. Confirmed occurrence: Rio de Janeiro. & $\begin{array}{l}\text { Couto et al., Phytotaxa, Vol. } 163 \text { n.4: } \\
\text { 229-234, } 2014 .\end{array}$ \\
\hline D. stegelmanniana R. Knuth & $\begin{array}{l}\text { Native. Endemic of Brazil Confirmed occurrence: Amazonas; Pará; Rondônia; } \\
\text { Roraima; Alagoas; Bahia; Pernambuco; Sergipe; Distrito Federal; Mato Grosso; } \\
\text { Espírito Santo; Minas Gerais; Rio de Janeiro; São Paulo; Paraná. }\end{array}$ & $\begin{array}{l}\text { Knuth, R., Notizbl. Königl. Bot. Gart. } \\
\text { Berlin, 7: 203, } 1917 .\end{array}$ \\
\hline D. stellaris R. Knuth & Native. Endemic of Brazil. Confirmed occurrence: Minas Gerais; Rio de Janeiro. & $\begin{array}{l}\text { Knuth, R., Pflanzenr. (Engler), IV, 43: } \\
\text { 233, } 1924 .\end{array}$ \\
\hline $\begin{array}{l}\text { D. stenophylla Uline ex R. } \\
\text { Knuth }\end{array}$ & $\begin{array}{l}\text { Native. Endemic of Brazil. Confirmed occurrence: Goiás; Minas Gerais. Possible } \\
\text { occurrence: Mato Grosso. }\end{array}$ & $\begin{array}{l}\text { Knuth, R., Nat. Pflanzenfam. [Engler \& } \\
\text { Prantl], Nachtr. 1: 84, } 1897 .\end{array}$ \\
\hline $\begin{array}{l}\text { D. subhastata Vell. cará-re- } \\
\text { dondo-chato }\end{array}$ & $\begin{array}{l}\text { Native. Confirmed occurrence: Alagoas; Bahia; Ceará; Paraíba; Pernambuco; } \\
\text { Sergipe; Espírito Santo; Minas Gerais; Rio de Janeiro; São Paulo; Paraná; Rio } \\
\text { Grande do Sul; Santa Catarina. }\end{array}$ & $\begin{array}{l}\text { Vellozo, J.M.C., Fl. Flumin. Icon., 10: t. } \\
\text { 121, } 1831 .\end{array}$ \\
\hline D. tauriglossum R. Knuth & Native. Endemic of Brazil. Confirmed occurrence: Alagoas; São Paulo & $\begin{array}{l}\text { Knuth, R., Pflanzenr. (Engler), IV, 43: } \\
\text { 350, } 1924 .\end{array}$ \\
\hline D. tenuiphyllum R. Knuth & Native. Endemic of Brazil. Confirmed occurrence: Mato Grosso. & $\begin{array}{c}\text { Knuth, R., Pflanzenr. (Engler), IV, 43: 76, } \\
1924 .\end{array}$ \\
\hline D. ternata Griseb. & $\begin{array}{l}\text { Native. Endemic of Brazil. Confirmed occurrence: Distrito Federal; Goiás; Es- } \\
\text { pírito Santo; Minas Gerais; Rio de Janeiro; Possible occurrence: Pará }\end{array}$ & $\begin{array}{c}\text { Grisebach, A., Vidensk. Meddel. Dansk } \\
\text { Natuhist. Foren. Kjøbenhavn, 1875: 158, } \\
1875 .\end{array}$ \\
\hline $\begin{array}{l}\text { D. therezopolensis Uline ex } \\
\text { R. Knuth }\end{array}$ & $\begin{array}{l}\text { Native. Endemic of Brazil. Confirmed occurrence: Espírito Santo; Minas Gerais; } \\
\text { Rio de Janeiro }\end{array}$ & $\begin{array}{l}\text { Knuth, R., Notizbl. Königl. Bot. Gart. } \\
\text { Berlin, 7: 211, } 1917 .\end{array}$ \\
\hline D. torticaulis R. Knuth & Native. Endemic of Brazil. Confirmed occurrence: São Paulo & $\begin{array}{l}\text { Knuth, R., Pflanzenr. (Engler), IV, 43: } \\
\text { 351, } 1924 .\end{array}$ \\
\hline D. trachyandra Griseb. & $\begin{array}{l}\text { Native. Endemic of Brazil. Confirmed occurrence: Goiás; Mato Grosso; Minas } \\
\text { Gerais. }\end{array}$ & $\begin{array}{c}\text { Grisebach, A., Vidensk. Meddel. Dansk } \\
\text { Natuhist. Foren. Kjøbenhavn, 1875: 155, } \\
1875\end{array}$ \\
\hline D. traillii R. Knuth & Native. Endemic of Brazil Confirmed occurrence: Amazonas & $\begin{array}{c}\text { Knuth, R., Pflanzenr. (Engler), IV, 43: 75, } \\
1924 .\end{array}$ \\
\hline $\begin{array}{l}\text { D. trifida L.f. Cará mimoso; } \\
\text { Cará roxo; Cará bola; Cará } \\
\text { rosado. }\end{array}$ & $\begin{array}{l}\text { Native. Endemic of South America. Confirmed occurrence: Acre; Amazonas; } \\
\text { Amapá; Pará; Rondônia; Tocantins; Maranhão; Paraíba; Pernambuco; Distrito } \\
\text { Federal; Goiás; Mato Grosso do Sul; Mato Grosso; Minas Gerais. Possible occur- } \\
\text { rence: Roraima. }\end{array}$ & LINNÉ FILIUS, C., Suppl. Pl. 427, 1782. \\
\hline D. trifoliata Kunth & Native. Confirmed occurrence: Acre & $\begin{array}{l}\text { Kunth, C.S., Nov. Gen. Sp. Pl., 1: 275, } \\
1816 .\end{array}$ \\
\hline D. trifurcata Hauman & Native. Confirmed occurrence: Rio de Janeiro; Rio Grande do Sul. & $\begin{array}{l}\text { Hauman, L.L., Anales Mus. Nac. Hist. Nat. } \\
\text { Buenos Aires, 27: 482, } 1916\end{array}$ \\
\hline D. trilinguis Griseb. & $\begin{array}{l}\text { Native. Endemic of Brazil. Confirmed occurrence: Minas Gerais; Rio de Janeiro; } \\
\text { São Paulo. }\end{array}$ & $\begin{array}{l}\text { Grisebach, A., Vidensk. Meddel. Dansk } \\
\text { Natuhist. Foren. Kjøbenhavn, 1875: 163, } \\
1875 .\end{array}$ \\
\hline
\end{tabular}




\begin{tabular}{|c|c|c|}
\hline D. trisecta Griseb. & $\begin{array}{c}\text { Native. Endemic of Brazil. Confirmed occurrence: Alagoas; Pernambuco; Distrito } \\
\text { Federal; Goiás; Minas Gerais; São Paulo; Paraná; Santa Catarina. }\end{array}$ & $\begin{array}{c}\text { Grisebach, A., Vidensk. Meddel. Dansk } \\
\text { Natuhist. Foren. Kjøbenhavn, 1875: 159, } \\
1875 .\end{array}$ \\
\hline $\begin{array}{c}\text { D. tubuliflora Uline ex R. } \\
\text { Knuth }\end{array}$ & Native. Endemic of Brazil. Confirmed occurrence: Santa Catarina. & $\begin{array}{c}\text { Knuth, R., Notizbl. Königl. Bot. Gart. } \\
\text { Berlin, 7: 189, 1917. }\end{array}$ \\
\hline $\begin{array}{c}\text { D. tubulosa Griseb. } \\
\text { D. warmingii (Uline ex R. } \\
\text { Knuth) R. Knuth }\end{array}$ & Native. Endemic of Brazil. Confirmed occurrence: Minas Gerais. & $\begin{array}{c}\text { Grisebach, A., Vidensk. Meddel. Dansk } \\
\text { Natuhist. Foren. Kjøbenhavn, 1875: 154, } \\
1875 .\end{array}$ \\
\hline D. wittiana R. Knuth & Native. Endemic of Brazil. Confirmed occurrence: Minas Gerais. & $\begin{array}{c}\text { Knuth, Pflanzenr. (Engler), IV, 43: 355, } \\
1924 .\end{array}$ \\
\hline
\end{tabular}

Note: Corroborating the data in the text and in the table, it is possible to identify that the most cultivated species in Brazil do not originate in the country.

\section{Conclusion}

The genus Dioscorea has many species without any scientific knowledge in the topics covered in this review, especially in the nutritional area and in other related topics, highlighting the species that synthesize phytohormones (steroidal saponins), as defense and protection strategies interfering in the metamorphosis of the insects or those that synthesize metabolites, with specific functions in the defense against microorganisms (fungi, bacteria and insects), viruses and toxins, used in hunting and fishing by indigenous peoples. All the reported applications showed that the Dioscorea species is a supplier of input for food, for the synthesis of hormones or for pharmacological and microbiological studies.

These applications are validated and based on the knowledge generated in scientific research and published in the articles available in the period mentioned and that show the importance of this knowledge for the food, industrial and pharmaceutical sector in Brazil. This review presents a collection of scientific data regarding the lack of scientific data on all species of confirmed occurrence in the country and public political to encourage the use and consumption of these species, which makes the genus neglected, making it impossible or hindering the efficiency in selfsustainable agricultural production and, as a consequence, limits the transformations in value-added products, generated by this gap of need for new scientific research.

\section{Acknowledgment}

This work was carried out with the support of the National Council for Scientific and Technological Development (CNPq), the Foundation for Research Support of the State of São Paulo (FAPESP), and the Environmental Research Institute of São Paulo

\section{References}

1. Peixoto NP, Lopes FJ, Caetano LC, Alencar LMC, Lemos EEP (2000) Inhame: 0 nordeste fértil. EDUFAL, Maceió, Brazil, p. 88.

2. Pedralli G (2002) Dioscoreaceae e araceae: Aspectos taxonômicos, etnobotânicos e espécies nativas com potencial para melhoramento genético. In: Simpósio Nacional sobre as Culturas do Inhame e do Taro, 2., João Pessoa, PB. Esclarecimentos sobre as denominações dos gêneros Dioscorea e Colocasia. pp: 37-53.

3. Leite RP, Nascimento LC, Oliveira MDM (2018) Inoculação de Curvularia eragrostidis em inhame (Dioscorea alata) cv. São Tomé. Summa Phytopathol 44(3): 281-282.
4. Moura RM, Oliveira IS, Torres GRC (2005) Doenças do inhame. In: Kimati (Ed.), Manual de Fitopatologia. 2 Doenças das Plantas Cultivadas. SP. Ceres, São Paulo, Brazil.

5. Ojelel S, Kakudidi EK (2015) Espécies de plantas comestíveis selvagens utilizadas por uma comunidade agrícola de subsistência no sub-condado de Obalanga, distrito de Amuria, Uganda. J Ethnobiol Ethnomed 11: 7.

6. Kirizawa M, Xifreda CC, Silva JH (2016) Diversidade florística de Dioscoreaceae na Reserva Biológica do Alto da Serra de Paranapiacaba, Santo André, São Paulo, Brasil. Hoehnea 43(1): 99-117.

7. Mesquita AS (2002) Inhame na Bahia: a produção no caminho da competitividade. In: Carmo CAS (Ed.), Inhame e taro: sistemas de produção familiar. Vitória: INCAPER, Brazil, pp: 33-49.

8. Opera LU (2003) YAMS: Post-harvest operation. In: Méjia D (Ed), Publishing FAO, New Zealand.

9. Egesi CN, Onyeka TJ, Asiedu R (2007) Gravidade das doenças antracnose e viral do inhame aquático (Dioscorea alata L.) na Nigéria: Efeitos do genótipo do inhame e data do plantio. Crop prot 26: 1259-1265.

10. Feijó JC, Cruz FGG, Melo RD, Rufino JPF, Damasceno JL (2016) Farinha de cará (Dioscorea trifida L.) sobre o desempenho, qualidade do ovo e bioquímica sérica de poedeiras comerciais leves. Rev Bras de Saú Prod Ani 17(3): 413-423.

11. Tavares WLS (2019) Farinha de Cará-roxo (Dioscorea trifida, L.) na alimentação de suínos na fase de terminação. 61 f. Dissertação (Mestrado em Ciência Animal)-Universidade Federal do Amazonas, Manaus, Brazil.

12. Siqueira MVBM (2009) Inhame (Dioscorea spp): uma cultura ainda negligenciada. Horticultura Brasileira 27: S4075-S4090.

13. Hsu SY, Hua KF, Lin CC, Lin CH, Hsu J, et al. (2004) Extract of Reishi polysaccharides induces cytokine expression via TRL4-modulated protein kinase signaling pathways. J Immunol 173: 5989-5999.

14. Castro AP, Fraxe TJP, Pereira HS (2012) Etnobotânica das variedades locais do cará (Dioscorea spp.) cultivados em comunidades no município de Caapiranga, estado do Amazonas. Acta Botanica Brasilica 26(3): 658667.

15. Viana CAS (2011) Plantas da Amazônia: Dioscoreaceae. Universidade de Brasília, Brasília, pp: 1079 -1094.

16. Foster S, Tyler VE (2000) Tyler's honest herbal: a sensible guide to the use of herbs and related remedies. ( $4^{\text {th }}$ edn), Haworth Herbal, New York, USA.

17. Hu K, Yao X (2002) The cytotoxicity of protoneodioscin (NSC-698789), a furostanol saponin from the rhizomes of Dioscorea collettii var. hypoglauca, against human cancer cells in vitro. Phytomedicine 9(6): 560-565.

18. Ramos ADS, Castro APD, Medeiro CM, Fraxe TDJP, Melo SRDD (2014) Avaliação da brotação para obtenção de mudas de diferentes partes 
do tubérculo de cará roxo (Dioscorea trifida L). Revista Brasileira de Agroecologia 9(1): 170-175.

19. Zárete NAH, Vieira MC, Mapeli NC, Siqueira AC (1998) Produção de clones de cará (Dioscorea spp.) em Dourados (MS). Anais Esc Agron e Vet 28(2): 117-121.

20. Leonel M, Cereda M (2002) Caracterização físico-química de algumas tuberosas amiláceas. Food Sci Technol 22(1).

21. Lopes PM (2016) Avaliação da atividade antimicrobiana de extratos de Dioscorea piperifolia frente a microrganismos patogênicos. 620 Dissertação (mestrado)- Universidade Federal do Rio de Janeiro, instituto de química, Programa de pós graduação em ciências de alimentos.

22. Barlagne C, Cornet D, Blazy JM, Diman JL, Ozier Lafontaine H (2017) Consumers' preferences for fresh yam: a focus group study. Food Science \& Nutrition 5(1): 54-66.

23. Trindade T, Soares LS, Furtado MC, Castro AA, Carnelossi MAG (2011) Composição centesimal de inhame (Dioscorea sp.) in natura e minimamente processado. Scientia Plena 7(6): 1-7.

24. Costa JCM, Costa BET (2016) Filmes comestíveis oriundos da fécula do cará (Dioscorea trifida).

25. Sousa GC, Pacheco FL, Reis NC, Teixeira AF (2019) Análisefísico-química e antioxidante do inhame (Dioscorea spp.). Revista Interdisciplinar Pensamento Científico 5(3): 30.

26. Dioscoreaceae in Flora do Brasil 2020 em construção (2020) Jardim Botânico do Rio de Janeiro.

27. Dong M, Feng XZ, Xiang B, Wu LJ (1999) Two novel furostanol saponins from the rhizomes of Dioscorea phantaica Prain et Burkil and their cytotoxic activity. Tetrahedron 57: 127-134.

28. Javier E, Salcedo J, Carlos G (2016) Functional Properties of Starch Yam (Dioscorea bulbífera, Dioscorea trifida, Dioscorea esculenta). Rev Téc Ing Univ Zulia 39(1): 1-2.

29. FAOSTAT DATA (2009) Food and Agriculture Organization.
30. FAOSTAT DATA (2010) Food and Agriculture Organization.

31. FAO, IFAD, UNICEF, WFP and WHO (2019) The state of food security and nutrition in the World 2019. Safeguarding against economic slowdowns and downturns. Rome, Italy.

32. Hu K, Al Jun Dong, Yao XS, Kobayashi H, Iwasak S (2012) A furostanol glycoside from rhizomes of Dioscorea colletti Var. Hypoglauca. Phytochemistry 44(7): 1339-1342.

33. Sanjeet K, Mahanti P, Singh NR, Rath SK, Jena PK, et al. (2017) Atividade antioxidante, potencial antibacteriano e caracterização da fração ativa do extrato de Dioscorea pentaphylla L. tuber coletado na Reserva da Biosfera Similipal, Odisha, India. Braz J Pharm Sci 53(4): e17006.

34. Machado PMM (2004) Estudo fitoquímico e biológico das folhas da Dioscorea Alata (Dioscoreaceae). 73 f. Dissertação (Mestrado) -Curso de Química de Compostos Bioativos, Universidade Federal de Pernambuco, Recife, Brazil.

35. Mabberley DJ (2008) Mabberley's plant-book a portable dictionary of plants, their Classifications, and Uses. ( $3^{\text {rd }}$ edn), Cambridge University Press, Cambridge, UK.

36. Martin FW (2020) The species of dioscorea containing sapogenin. Economic Botany 23(4): 373-379.

37. Mulholland DA (2002) The isolation of the Amaryllidaceae alkaloid crinamine from Dioscorea dregeana (Dioscoreaceae). Biochemical systematics and ecology 30(2): 183-185.

38. Narula A, Kumar S, Bansal KC, Srivastava PS (2007) In vitro micropropagation, differentiation of aerial bulbils and tubers and diosgenin content in Dioscorea bulbifera. Planta Med 69(8): 778-779.

39. Pedralli G (2002) Distribuição geográfica e taxonômica das famílias Araceae e Dioscoreaceae no Brasil. In: Carmo CAS, (Ed.), Inhame e taro: sistemas de produção familiar. Vitória, ES: INCAPER, pp: 15-26.

40. Reis R, Aacheri DPR, Devilla I (2010) Propriedades Físicas do Tubérculo e Propriedades Químicas e Funcionais do Amido de Inhame (Dioscorea sp.) Cultivar São Bento. Revista Agrotecnologia-Agrotec 1: 71-88. 